\title{
Diffusion and the formation of vorticity staircases in randomly strained two-dimensional vortices
}

\author{
MATTHEW R. TURNER ${ }^{1} \dagger$, ANDREW P. BASSOM ${ }^{2}$ \\ AND ANDREW D. GILBERT $T^{1} \ddagger$ \\ ${ }^{1}$ Mathematics Research Institute, School of Engineering, Computing and Mathematics, \\ University of Exeter, Exeter EX4 4QF, UK \\ ${ }^{2}$ School of Mathematics and Statistics, University of Western Australia, Crawley 6009, Australia
}

(Received 9 October 2008; revised 22 June 2009; accepted 22 June 2009)

The spreading and diffusion of two-dimensional vortices subject to weak external random strain fields is examined. The response to such a field of given angular frequency depends on the profile of the vortex and can be calculated numerically. An effective diffusivity can be determined as a function of radius and may be used to evolve the profile over a long time scale, using a diffusion equation that is both nonlinear and non-local. This equation, containing an additional smoothing parameter, is simulated starting with a Gaussian vortex. Fine scale steps in the vorticity profile develop at the periphery of the vortex and these form a vorticity staircase. The effective diffusivity is high in the steps where the vorticity gradient is low: between the steps are barriers characterized by low effective diffusivity and high vorticity gradient. The steps then merge before the vorticity is finally swept out and this leaves a vortex with a compact core and a sharp edge. There is also an increase in the effective diffusion within an encircling surf zone.

In order to understand the properties of the evolution of the Gaussian vortex, an asymptotic model first proposed by Balmforth, Llewellyn Smith \& Young (J. Fluid Mech., vol. 426, 2001, p. 95) is employed. The model is based on a vorticity distribution that consists of a compact vortex core surrounded by a skirt of relatively weak vorticity. Again simulations show the formation of fine scale vorticity steps within the skirt, followed by merger. The diffusion equation we develop has a tendency to generate vorticity steps on arbitrarily fine scales; these are limited in our numerical simulations by smoothing the effective diffusivity over small spatial scales.

\section{Introduction}

There has been much study of the evolution of passive scalars in fluid flows. In some cases, chosen either for mathematical convenience or with a certain application in mind, the fluid flow is taken to be kinematic while in other instances it is obtained dynamically through full simulation of the Navier-Stokes equations. Problems involving active scalars, in which the scalar feeds back on the flow field, are much less well understood. In particular, within two-dimensional fluid flows the

$\dagger$ Present address: Environment and Technology, University of Brighton, Lewes Road, Brighton BN2 4GJ, UK.

$\ddagger$ Email address for correspondence: A.D.Gilbert@exeter.ac.uk 
vorticity becomes a scalar which is subject to mixing by the underlying fluid flow, but at the same time it specifies the flow. It is then a subtle interaction of fluid stability and mixing properties that determines the evolution of such systems.

Here the aim is to study this interaction and the subsequent diffusive evolution in a simple two-dimensional geometry which is broadly relevant to geophysical applications. Our chosen model is a coherent vortex in an external irrotational flow field, such as might be generated by the motion of other coherent vortices in two-dimensional turbulence, or by the motion of boundaries. If a two-dimensional vortex, for simplicity say a Gaussian vortex, is subjected to a short period of weak spatially uniform external strain, the effect is to distort the vortex and generate a spiral structure of vorticity pulled out by the differential rotation of the vortex. This is a mode with azimuthal wavenumber $m=2$, and enstrophy, which is inviscidly conserved, is transferred from the mean to the mode. This process was studied in detail by Bassom \& Gilbert $(1998,1999)$ who identified a number of distinct regimes. First is a dynamical feedback (or 'rebound') whereby the enstrophy transfers from the $m=2$ mode to the mean: the core of the vortex dynamically returns to axisymmetry, so suppressing the generation of fluctuations and mixing there. Although this behaviour was noted by Bassom \& Gilbert $(1998,1999)$, it was not until the work of Schecter et al. (2000) and Balmforth, Llewellyn Smith \& Young (2001) that it could be claimed to be well understood. These latter authors used an asymptotic model to link the suppression of fluctuations to the existence of a so-called 'quasi-mode' in the vortex. Properties of quasi-modes are a combination of those of continuous spectrum with others of a normal mode, including an exponential decay rate or Landau pole, first discussed in the fluid context by Briggs, Daugherty \& Levy (1970) and more recently in general boundary layer flows by Shrira \& Sazonov (2001).

Leaving aside the technicalities of quasi-modes, the key point is that there is a dynamical response of the vortex which has the effect of suppressing mixing in the core of the vortex. In many ways the core of the vortex has a damped 'elastic' response, as we discuss further below, which is a function of the vorticity profile. However the behaviour of the external strain is itself to modify the profile, and change the response to future strain. This can be studied in a deterministic context by applying strain fields, and mapping out the dynamical response and the resulting modified profiles: papers that discuss this include Balmforth et al. (2001) and Turner $\&$ Gilbert $(2007,2009)$. In the present work the intention is to determine the effect of a continuous external forcing, so that the vortex spreads and evolves, changing its response as it does so. If the external strain is weak, the spreading occurs on a slow time scale, and the response at any instant is given by a problem which is a linearization about the profile at that time. If the external forcing is also random, with a given correlation function, a weakly nonlinear theory can be used to derive a diffusion equation that governs the spread of the vortex. Since this spreading involves the response of the vortex to each frequency in the external strain, and each response depends on the whole profile, the diffusion equation on the long time scale is both nonlinear and non-local. Although it cannot be written down in an explicit form, it may be simulated numerically and its properties explored.

In the coming section we investigate the particular case of a Gaussian vortex placed in a random strain field: our computations demonstrate the suppressed diffusion in the core and the enhanced mixing further out in the tails, together with a number of other phenomena including the formation of vorticity staircases, with steps and transport barriers. To confirm these findings, and to obtain more detail and greater understanding of our results, we then consider the system of equations in 
Balmforth et al. (2001). These equations model the interaction of a normal mode riding on a compact central vortex, with a surrounding 'skirt' of weak vorticity. This is a good qualitative model of a Gaussian vortex and we find similar phenomena as for the Gaussian case.

A number of other studies have focused on the interaction of the dynamical effects of vorticity and the mixing properties of the flow within a deterministic setting. Haynes, Poet \& Shuckburgh (2007) compare the transport in a kinematically prescribed flow modelling a meandering jet, with a similar flow on a beta plane under topographic forcing. In both cases the presence of barriers inhibits mixing: however in the dynamical case there are significant changes in the flow field when a barrier is broken and vorticity is homogenized in a large area of the flow. Perhaps the closest studies to the present paper are del Castillo Negrete $(2000 a, b)$ : these papers consider a variety of models in which advected scalars change the parameters of the flow, or a mapping such as the standard map (see also Boffetta et al. 2003). In the model closest to ours, the 'single wave model', vorticity is advected by a shear flow whose profile is chosen so that it possesses a neutral large-scale mode. The mode amplitude interacts with the vorticity distribution to give a simplified system similar to that derived by Balmforth et al. (2001). The presence of the neutral mode means that the flow tends to develop a critical layer containing cat's eyes and the mixing of vorticity within these eyes can have a strong effect on the flow field: for example introducing weak vorticity into the eyes can lead to resonant oscillations and enhanced mixing (del Castillo Negrete 2000a).

The process of vorticity mixing in the presence of a neutral mode (del Castillo Negrete $2000 a, b$ ) is very relevant to our study of a Gaussian vortex, even though this profile possesses no neutral modes (Briggs et al. 1970). The reason is that, in a sense we will explain, the Gaussian vortex 'nearly' has a neutral mode. This is the approach taken in Balmforth et al. (2001), who break a Gaussian vortex into a compact coherent core, for example a top-hat or Rankine vortex, surrounded by a tail or skirt of weak vorticity. A Rankine vortex supports a neutral $m=2$ normal mode (or Kelvin wave). This is undamped, but if a weak vorticity gradient is introduced at the radius where fluid particles corotate with the wave, i.e. in the critical layer, Balmforth et al. (2001) show how this can stabilize or destabilize the wave, depending on the sign of the gradient. In a similar way, for qualitative purposes a Gaussian vortex can be treated as having a core that supports a neutral mode, but which is damped by vorticity at the outer edges of the vortex: this combination forms a quasi-mode in the linear regime giving the damped elastic behaviour mentioned above. If pushed hard enough cat's eyes are created in the critical layer, with a profile modified sufficiently for a true neutral normal mode to exist on the vortex (Balmforth et al. 2001; Turner \& Gilbert 2007).

These earlier studies have focused on the initial value problem, with linear behaviour such as quasi-mode damping, and the nonlinear generation of cat's eyes beyond some amplitude threshold. Our study here is distinguished by using a weak random external forcing so that the vortex is always in a linear regime, and by using a weakly nonlinear expansion to set up a diffusion equation for the vorticity profile on a long time scale. A related study was presented by two of the present authors a decade ago (Bassom $\&$ Gilbert 1999). There we again derived a weakly nonlinear system for the evolution of the mean profile, and proceded to simulate it with a realization of the random forcing included explicitly. Although some results were obtained, for example the demonstration that the suppression of vorticity arising from the quasi-mode has the effect of decreasing the spread of the vortex compared to a passive scalar, it was 
not possible to follow the system very far in time. The present study, which can be considered a more advanced version of Bassom \& Gilbert (1999), as the random component is ensemble averaged at the outset, will highlight some of the limitations of that earlier work, in particular our present finding that the interaction of dynamical and diffusive properties of vorticity in these systems leads to small-scale instability and the generation of fine scale steps in the vorticity profiles.

The remainder of the paper is structured as follows. In $\S 2$ we consider the case of the Gaussian vortex in a random strain field: a diffusion equation is derived for the evolution of the profile on a long time scale and numerical experiments are performed. We attempt to throw more light on the processes involved by examining the model of Balmforth et al. (2001) in $\S 3$. In these sections theory is developed, followed by numerical simulations, but technical details are relegated to three appendices. Finally, $\S 4$ offers some concluding discussion.

\section{Gaussian vortex in random strain}

\subsection{Analytical development}

We use the equations for inviscid planar flow written in the form

$$
\partial_{t} \omega=J(\psi, \omega), \quad \omega=-\nabla^{2} \psi,
$$

with the velocity field $\boldsymbol{u}=\left(r^{-1} \partial_{\theta} \psi,-\partial_{r} \psi\right)$ in plane polar coordinates $(r, \theta)$ and

$$
r J(a, b) \equiv\left(\partial_{r} a\right)\left(\partial_{\theta} b\right)-\left(\partial_{\theta} a\right)\left(\partial_{r} b\right) .
$$

We begin with an axisymmetric vortex, and adopt non-dimensional units based on its width and circulation. For example, our initial condition of a Gaussian vortex is

$$
\omega=\bar{\omega}=(4 \pi)^{-1} \mathrm{e}^{-r^{2} / 4},
$$

where $\bar{\omega}$ is the mean vorticity profile, averaged over $\theta$. With a general vorticity profile is an associated mean stream function $\bar{\psi}$, angular velocity $\alpha$ and vorticity gradient $r \beta$ given by

$$
\bar{\omega}=-r^{-1} \partial_{r}\left(r \partial_{r} \bar{\psi}\right), \quad \alpha=-r^{-1} \partial_{r} \bar{\psi}, \quad \beta=r^{-1} \partial_{r} \bar{\omega} .
$$

We now impose a weak, external strain field on the vortex by requiring that

$$
\psi(r, \theta, t) \sim(-2 \pi)^{-1} \log r+\varepsilon q(t) r^{m} \mathrm{e}^{\mathrm{i} m \theta}+\text { c. c. } \quad(r \rightarrow \infty),
$$

where c.c. denotes the complex conjugate. This is the monopolar field from the vortex plus the $\mathrm{e}^{ \pm \mathrm{i} m \theta}$ components of a general multipolar field of complex amplitude $\varepsilon q(t)$ with $0<\varepsilon \ll 1$. The resulting flow is irrotational outside the vortex, at radii where $\omega$ is effectively zero. We will focus on the case $m=2$ numerically, that of spatially uniform external strain, but leave the general value of $m$ in the mathematical development for clarity. (We note that $m=1$ gives a translation, while we expect mixing properties for modes $m>2$ to be similar to $m=2$.)

The time dependence of the statistically stationary external flow is given by the correlation function of $q(t)$ and we shall consider two cases. The first of these is a flow that is delta correlated in time so that

$$
\left\langle q(t) q^{*}\left(t^{\prime}\right)\right\rangle=\delta\left(t-t^{\prime}\right), \quad w(p)=1 .
$$

Here the brackets $\langle\cdot\rangle$ denote an ensemble average and $w(p)$ is a corresponding weight function which we note here for reference and which emerges in computing the feedback on the mean profile in Appendix A. Physically, this function $w(p)$ describes 
the contribution to frequency $p$ from the random function $q(t)$. Our second example has a peak at a rotational frequency $b$ and an exponential fall-off

$$
\left\langle q(t) q^{*}\left(t^{\prime}\right)\right\rangle=\frac{a}{2} \mathrm{e}^{-a\left|t-t^{\prime}\right|} \mathrm{e}^{-\mathrm{i} m b\left(t-t^{\prime}\right)}, \quad w(p)=\frac{a^{2}}{(p-m b)^{2}+a^{2}},
$$

so that the weight is largest around frequencies $p=m b$. The delta correlated case is recaptured in the limit $a \rightarrow \infty$ for any fixed $b$.

Now consider the evolution of the flow and vorticity field, for example starting from the Gaussian initial condition (2.2). As time proceeds so the external flow generates fluctuations, i.e. components proportional to $\mathrm{e}^{ \pm \mathrm{i} m \theta}$ in the vorticity field. For small $\varepsilon$ these are weak and governed by the equation for vorticity, linearized about the mean profile $\bar{\omega}$. There is then a feedback, of magnitude $\varepsilon^{2}$, from the quadratic terms, which leads to a slow drift of the mean profile $\bar{\omega}$, on a long time scale.

To obtain the equation for this drift, we begin by taking $\varepsilon \ll 1$ in the external strain (2.4), and introduce a long time scale $\tau=\varepsilon^{2} t$ together with a weakly nonlinear expansion

$$
\begin{aligned}
& \omega(r, t, \tau)=\bar{\omega}(r, \tau)+\varepsilon \hat{\omega}(r, t, \tau) \mathrm{e}^{\mathrm{i} m \theta}+\text { c. c. }+\cdots, \\
& \psi(r, t, \tau)=\bar{\psi}(r, \tau)+\varepsilon \hat{\psi}(r, t, \tau) \mathrm{e}^{\mathrm{i} m \theta}+\text { c. c. }+\cdots .
\end{aligned}
$$

Dropping the hats yields equations for the fluctuating vorticity and stream function

$$
\partial_{t} \omega+\mathrm{i} m \alpha \omega+\mathrm{i} m \beta \psi=0, \quad-\omega=\left(\partial_{r}^{2}+r^{-1} \partial_{r}-r^{-2} m^{2}\right) \psi .
$$

These evolve in the angular velocity field and background vorticity gradient given in (2.3) in terms of the mean profile. On the longer, $\tau$ time scale, the mean profile obeys

$$
\partial_{\tau} \bar{\omega}+r^{-1} \partial_{r}(r F)=0, \quad F=2 m r^{-1} \operatorname{Im}\left\langle\omega \psi^{*}\right\rangle,
$$

where the angled brackets denote an ensemble average over realizations of the random forcing, and also an average over evolution on the fast $t$ time scale.

Our aim is to solve the fluctuating problem and then derive the feedback on the mean profile, in a form suitable for numerical time stepping. We use a Laplace transform approach and define

$$
\widetilde{f}(p)=\int_{0}^{\infty} \mathrm{d} t \mathrm{e}^{\mathrm{i} p t} f(t), \quad f(t)=-\frac{1}{2 \pi} \int_{\Gamma} \mathrm{d} p \mathrm{e}^{-\mathrm{i} p t} \widetilde{f}(p) .
$$

Here $\Gamma$ is a contour taken from $+\infty$ to $-\infty$ above all singularities of the function $\widetilde{f}(p)$. From (2.9), in Laplace transform space we have to solve

$$
\widetilde{\omega} \equiv-\left(\partial_{r}^{2}+r^{-1} \partial_{r}-r^{-2} m^{2}\right) \widetilde{\psi}=\frac{\beta(r)}{p / m-\alpha(r)} \widetilde{\psi}
$$

for $\widetilde{\psi}(r, p)$ with

$$
\widetilde{\psi}(r, p) \sim r^{m} \widetilde{q}(p) \quad \text { as } \quad r \rightarrow \infty
$$

and $\widetilde{\psi}(0, p)=0$. We formally write the solution as

$$
\widetilde{\psi}(r, p)=M(r, p) \widetilde{q}(p), \quad \widetilde{\omega}(r, p)=N(r, p) \widetilde{q}(p) .
$$

As discussed by Briggs et al. 1970, there is a singularity whenever the external frequency $p=m \alpha(r)$ for some resonant radius $r$ and the behaviour near such points is relevant to us. 
Suppose we consider a fixed value $p=m \alpha(s)$ for some $s$, and vary $r$; then the solution to the above differential system has the following behaviour as $r \rightarrow s$. The leading order singular term for the vorticity is a simple pole

$$
N(r, p)=-C(p) \mu(s)(r-s)^{-1}+\cdots
$$

and for the stream function is

$$
M(r, p)=C(p)[1+\mu(s)(r-s) \log (s-r)+\cdots]+D(p)[(r-s)+\cdots],
$$

for $r<s$, while

$$
M(r, p)=C(p)[1+\mu(s)(r-s)(\log (r-s)+i \chi)+\cdots]+D(p)[(r-s)+\cdots]
$$

for $r>s$. The coefficients $C$ and $D$ depend on $p$ and are fixed by the boundary conditions at $r=0$ and infinity. The quantity $\mu(s) \equiv \beta(s) / \partial_{s} \alpha(s)$ while the angle $\chi$ is a phase shift which is chosen to select the correct branch of the logarithmic singularity in $\widetilde{\psi}$. We have in mind letting the point $p$ approach the real axis from above which means that for $\partial_{s} \alpha(s)>0$ the point $s$ also approaches from above and the phase shift is $\chi=\pi$. On the other hand, if $\partial_{s} \alpha(s)<0$ (as in the Gaussian vortex), $s$ approaches from below and $\chi=-\pi$.

This gives a formal solution to the linear problem for the fluctations driven by any external frequency $p$. It then remains to calculate the feedback on the mean profile by calculating the average $\left\langle\omega \psi^{*}\right\rangle$ in (2.10). We relegate this calculation to Appendix A and give only the final result here, which is a diffusion equation for $\bar{\omega}(r, \tau)$,

$$
\partial_{\tau} \bar{\omega}+r^{-1} \partial_{r}(r F)=0, \quad F(r)=-\kappa(r) \partial_{r} \bar{\omega},
$$

with a radial vorticity flux $F$ and an effective diffusivity taking the form

$$
\kappa_{*}(r)=m^{2} r^{-2}|C(p)|^{2} w(p), \quad p \equiv m \alpha(r) .
$$

In (2.18) and (2.19) as used for simulations, $\kappa$ and $\kappa_{*}$ are not quite the same: $\kappa$ is a smoothed version of $\kappa_{*}$ in a sense that we will clarify shortly. We note that the quantities $\alpha, \kappa_{*}, F$ and $C$ all also change with $\tau$ as the underlying profile $\bar{\omega}$ evolves but we suppress this dependence for brevity: only the specification of the random forcing $w(p)$ is independent of $\tau$. Instead it is more useful to emphasize the dependence of quantities on radius $r$ or frequency $p$ as appropriate.

The content of (2.19) is worthy of some comment. At a given radius $r$, there is a crucial link to a frequency $p$ via the angular velocity $\alpha(r)$ : it is at this frequency that the external forcing is resonant with the motion of fluid elements, accounting for the weighting factor $w(p)$. The quantity $C(p)$ controls the transport and is the strength in (2.15) of the singularity at the resonant radius $r$ of the linear solution driven by external forcing of frequency $p$. Computing $C(p)$ has to be done numerically for each radius $r$ and depends on the whole vorticity profile, through integration of the ordinary differential equation (2.12) involving the angular velocity $\alpha$ and vorticity gradient $r \beta$, linked in turn to $\bar{\omega}$. The effective diffusivity $\kappa_{*}(r)$ is always non-negative and the resulting diffusion equation is nonlinear and non-local, inheriting these properties from the Euler equation. These features make further analytical progress difficult, at least in any general case, but can be dealt with numerically.

We also mention the case of a passive scalar field $\sigma$. If we take a flow field with a fixed angular velocity $\alpha(r)$ and simply add on the non-axisymmetric component of the random strain field (2.4) to obtain a prescribed purely kinematic flow field, the 
(a)

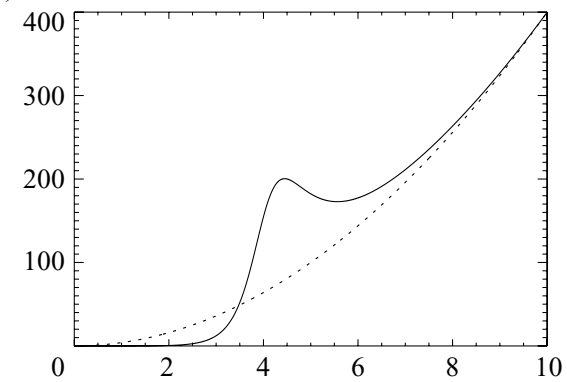

(b)

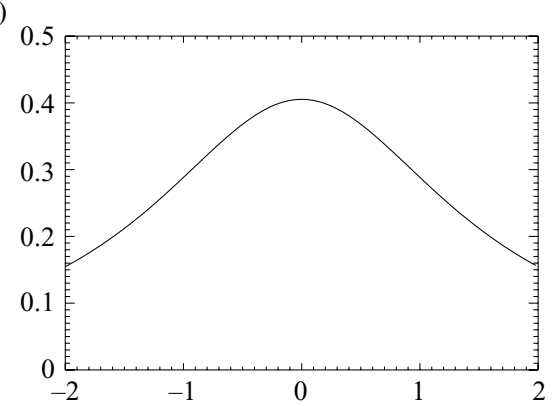

FIGURE 1. Effective diffusivity $(a) \kappa(r)$ plotted for the Gaussian vortex at $\tau=0$ (with the scalar diffusivity shown dotted), and (b) $\kappa(y)$ plotted for the model of Balmforth et al. (2001) at $\tau=0$ $($ see $\S 3)$.

resulting radial scalar flux is $F=-\kappa_{\text {scalar }}(r) \partial_{r} \bar{\sigma}$ with

$$
\kappa_{\text {scalar }}(r)=m^{2} r^{-2} r^{2 m} w(p), \quad p \equiv m \alpha(r) .
$$

This is in agreement with results given in Bassom \& Gilbert (1999), up to normalization. For large $r, C(p) \simeq r^{m}$ and so this is also the large- $r$ form of the effective diffusivity (2.19).

Notice that in the delta correlated case $w(p)=1$, the scalar effective diffusivity (2.20) has no dependence whatsoever on the axisymmetric component of the flow field. This is a result of the limit of delta correlation: the external flow changes so rapidly that the advection by the axisymmetric flow component is irrelevant. This is not the case for the fully dynamical vorticity problem: even in the delta correlated case, the profile enters into the quantity $C(p)$ in (2.19). In some loose sense, the behaviour of the vortex introduces a 'memory' into the problem: if a blob of passive scalar is moved to a different radius there is no dynamical response, whereas for a blob of vorticity the global flow field continues to evolve on its own time scale: this is the rebound phenomenon of Bassom \& Gilbert (1999), discussed in the introduction and now understood as quasi-mode damping.

Figure 1( $a)$ shows the effective diffusivity $\kappa(r)$ in $(2.19)$ for the Gaussian profile, at $\tau=0$. (Figure $1 b$ shows the analogous function (3.16) for the model of Balmforth et al. 2001 discussed later in $\S 3$ ). This has a number of interesting features. The corresponding scalar diffusivity (2.20) is shown dotted, and the two coincide for large $r$. Near the origin, say for $r \leqslant 3$, the vorticity diffusivity is much smaller than the scalar diffusivity: this is a consequence of the elastic behaviour of the vortex core discussed in the introduction. An external frequency that is in the range to excite fluctuations in the core of the vortex will be met with a wave-like response and no net transport. However further out, at $r \simeq 4, \kappa(r)$ is rather larger than the scalar diffusivity: here the external forcing meets a large response from the vortex, essentially exciting the quasi-mode and giving strong mixing in the critical layer.

\subsection{Numerical results}

We constructed a code to follow the vorticity on the long $\tau$ time scale. Starting with the profile (2.2) the code integrates the diffusion equation defined by (2.18) and (2.19); $\alpha$ is computed from $(2.3), C(p)$ is obtained from $(2.12-2.17)$ and we used the delta correlated case $w(p)=1$ in all our computations (other choices give similar results). The details of the numerical method are discussed in Appendix B. 
If the equations are solved with no smoothing at all (so that $\kappa \equiv \kappa_{*}$ ), it turns out that the results are sensitive to the radial grid used, from $r=0$ to $r=R$ : the finer the grid, the closer the steps that form initially. Therefore, to obtain numerical results that have a clear mathematical setting, i.e. independent of resolution, we impose a cap on the values of the diffusivity and smooth the diffusivity over a small length scale. Specifically, once we have computed $\kappa_{*}(r)$ from $(2.19)$, we replace it by

$$
\kappa=\kappa_{\text {scalar }} G_{\delta} F_{\delta}\left(\kappa_{*} / \kappa_{\text {scalar }}\right) .
$$

Here we first normalize $\kappa$ using the scalar diffusivity, which grows with a power law dependence in (2.20). The first operation then caps this at a level $\delta^{-1}$,

$$
F_{\delta}(s)=\delta^{-1} \tanh (s \delta),
$$

and the second smooths it over a scale of order $\delta$,

$$
\left(G_{\delta} f\right)(s)=\pi^{-1 / 2} \delta^{-1} \int_{-\Lambda \delta}^{\Lambda \delta} \mathrm{e}^{-\left(s-s^{\prime}\right)^{2} / \delta^{2}} f\left(s^{\prime}\right) \mathrm{d} s .
$$

This smoothing is taken over $\Lambda=3$ standard deviations of the Gaussian, and is done numerically from $r=\Lambda \delta$ to $r=R-\Lambda \delta$ : this covers all the radii at which fine structure develops, and structure in the diffusivity and vorticity profile is eliminated at scales smaller than $\delta$. We discuss this smoothing further below.

The results of runs for the Gaussian initial condition are shown in figures 2 and 3: the panels of figures 2 and 3 are distinguished by different values of the smoothing parameter $\delta$. Figure $2(a)$ has the largest smoothing parameter, $\delta=0.05$ : reading up the curves shows the evolution of the profile. The Gaussian tail breaks up into a single step, which then disappears to leave a sharp-edged vortex. In figure $2(d)$ the evolution of the logarithm of the diffusivity $\log \kappa(r)$ is shown: it may be seen that a double peak emerges; the first peak, around $r=3$, corresponds to the flat part of the step and the second peak lies just beyond the edge of the sharpening vortex. The two peaks then merge and move inwards, in concert with the sharpening edge of the vortex. Another view of our results with this choice of $\delta$ is shown in figure $3(a, d)$. Figure $3(a)$ depicts the evolution of the vorticity gradient $\partial_{r} \bar{\omega}(r, \tau)$ and figure $3(d)$ depicts the effective diffusivity $\kappa(r, \tau)$ in space-time or 'butterfly' diagrams. Figure 3(a) shows the initial formation of a single vorticity step (with also some outgoing ripples), followed by its sudden evaporation to leave a sharp-edged vortex. The behaviour of the vorticity gradient is linked to the effective diffusivity (figure $3 d$ ).

The evolution we see in figures $2(a, d)$ and $3(a, d)$ is the result of a runaway process in which the effective diffusivity rapidly increases at certain radii. Initially the behaviour of the vortex is roughly that of a damped elastic mode, the quasi-mode. The damping arises from the tail of the Gaussian vortex and limits the response to the external random strain, giving the effective diffusivity plotted in figure $1(a)$, with a peak around $r \simeq 4$. This peak has the effect of flattening the profile around $r \simeq 4$ by diffusion, leading to less damping of the quasi-mode and so further enhancement of the effective diffusivity (the peak also moves inwards because of the modified profile). This process rapidly diffuses the vorticity out from the edge of the vortex, to leave a sharp-edged vortex and high effective diffusivity outside. We can characterize this final state as a coherent vortex with a very clear edge, surrounded by what is often described as a 'surf zone' of enhanced diffusion (McIntyre \& Palmer 1984).

As we reduce $\delta$, increasingly fine scale structure appears in our runs. In figure $2(b, c)$ we see a more complex picture emerging, in which the profile develops fine scale 
(a)



(b)

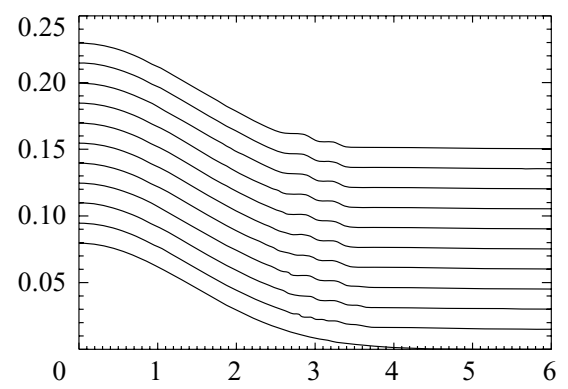

(c)



(d)

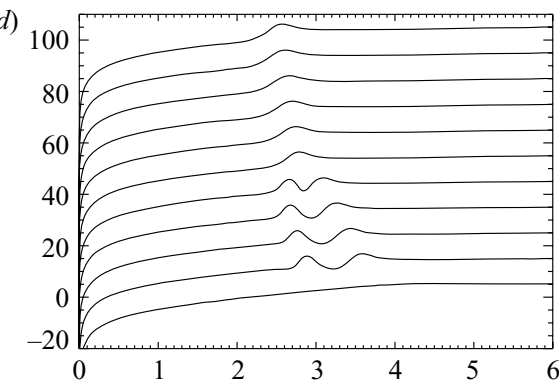

(e)

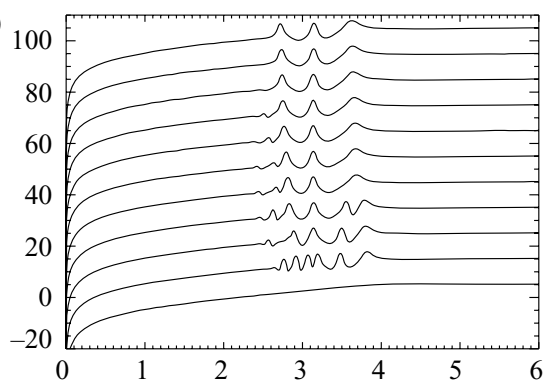

(f)

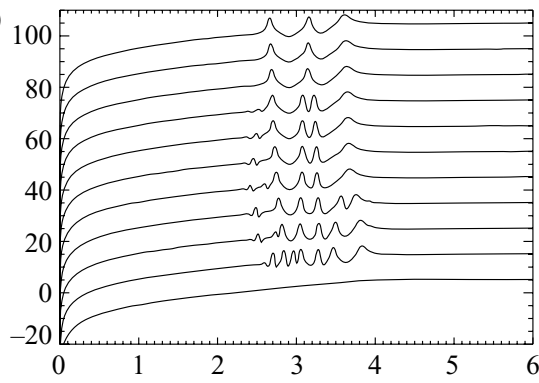

Figure 2. Evolution of a Gaussian vortex with smoothing $(a, d) \delta=0.05,(b, e) \delta=0.02$ and $(c, f) \delta=0.015$. The curves show a sequence of vorticity profiles $\bar{\omega}(a-c)$ and corresponding effective diffusivities $\log \kappa(d-f)$, plotted against $r$. In each panel the curves are separated by additive constants and given in steps of $(a, d) 0.02$ and $(b, c, e, f) 0.002$ of $\tau$, reading up the curves.

steps initially (with outgoing ripples), followed by merger. Where the profile is flat the effective diffusivity takes very large values, and where the profile shows sharp gradients, the diffusivity is suppressed. We can think of the vertical regions as barriers, where reduced diffusion inhibits transport, surrounding well-mixed regions. We note that there appears to be a limited range of radii where steps form initially, for the smaller values of $\delta$ used. The reason for this became apparent in studying the model of Balmforth et al. (2001), and we give numerical results for this in the next section, with some analytical justification in Appendix C. In this model, with $\delta=0$, there is a range of radii for which a fine scale instability can occur: a weak small-scale perturbation, wavenumber $k \gg 1$, on the vorticity profile has a growth rate proportional to $k^{2}$. This effect, which is natural given the flattening process described above, gives results sensitive to grid scale, no matter what the numerical scheme. The same effect appears to occur for the evolution of the Gaussian vortex and is the reason for the introduction of the smoothing parameter $\delta$. 
(a)
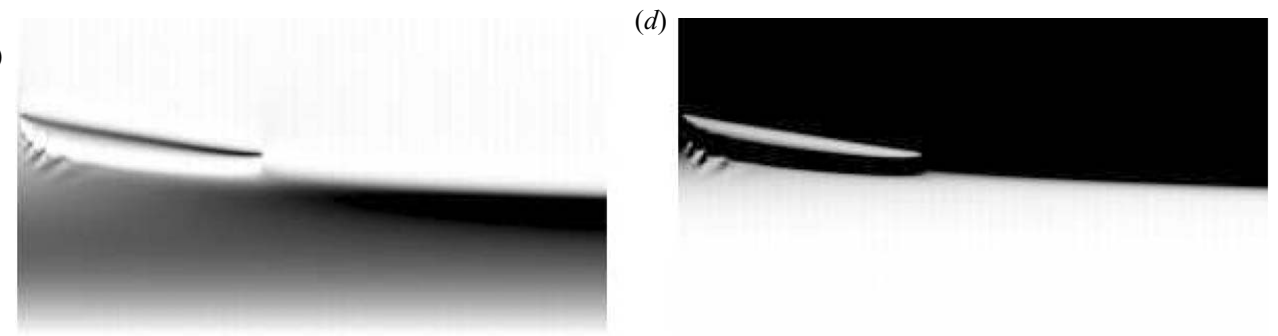

(b)


$(f)$

(c)
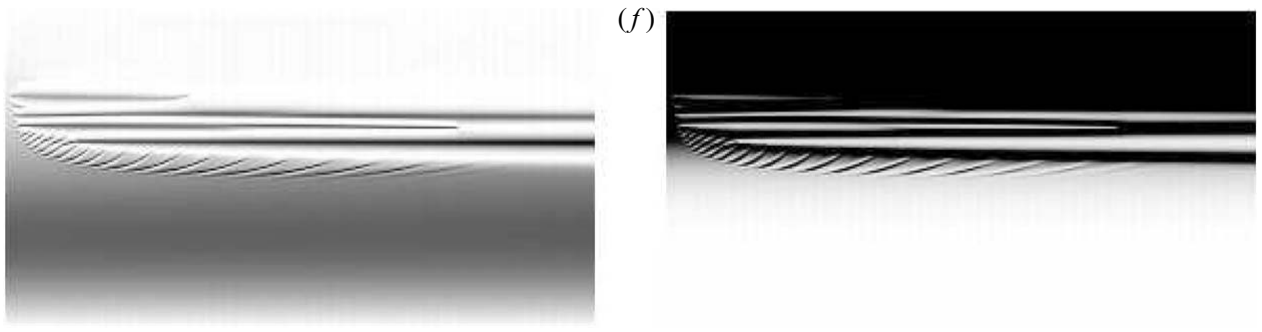

FIGURE 3. Space-time diagram of the evolution of the vorticity gradient $-\partial_{r} \bar{\omega}(r, \tau)(a-c)$ and effective diffusivity $\kappa(r, \tau)(d-f)$, plotted in grey scales in the $(\tau, r)$ plane for $(a, d) \delta=0.05$, $0 \leqslant \tau \leqslant 0.2(b, e) \delta=0.02,0 \leqslant \tau \leqslant 0.02$ and $(c, f) \delta=0.015,0 \leqslant \tau \leqslant 0.02$. The grey scale coding is capped at levels $-\partial_{r} \bar{\omega}=0.05, \kappa=10$, corresponding to black; zero is white. The vertical range is $0 \leqslant r \leqslant 5$ and $\tau$ runs horizontally.

We stress that our results are dependent on the choice of the smoothing parameter, and this requires some discussion and interpretation. Certainly introducing a smoothing effect in this way is not ideal, and the instabilities observed strictly show that the multiple scale framework is breaking down, the separation of time scales being lost. However smoothing the distribution of $\kappa$ is physically natural. The point is that in our weakly nonlinear framework there is no mechanism to saturate the response to an external frequency if the vortex profile develops a neutral normal mode. However in the original unscaled problem nonlinearity will step in and limit the excursions of fluid particles. In fact, if a vortex is subjected to a single frequency $p$ for a long time, there follows the development of cat's eyes of a width $\varepsilon^{1 / 2}$ in the vortex profile. This is the width of the resonance, limited by the local gradient of angular velocity (which incidentally does not generate the same fine scales as the vorticity profile). It is thus natural to limit $\kappa$ to be smooth over scales $\delta=O\left(\varepsilon^{1 / 2}\right)$. Similarly the diffusivity is dimensionally $\mathscr{L} \mathscr{U}=O\left(\varepsilon^{3 / 2}\right)$ for motion across a cat's eye of width $\mathscr{L}=O\left(\varepsilon^{1 / 2}\right)$ with velocity $\mathscr{U}=O(\varepsilon)$ : however this is on the short $t$ time scale and corresponds to a limit $\kappa=O\left(\varepsilon^{-1 / 2}\right)$ on the long $\tau$ time scale.

In short it is natural to introduce a parameter $\delta$ of order $\varepsilon^{1 / 2}$ and to use it to smooth the effective diffusivity over scales $\delta$ and cap at values $\delta^{-1}$. We normalize by the scalar diffusivity in order not to cap the algebraic growth of $\kappa$ for large $r$, which 
is reasonable as this is the true effect of random strain, with its growing velocity field as $r \rightarrow \infty$. As we do not have any more systematic way to estimate $\delta$ and its dependence on radius and time, we have taken it to be constant for each run and explored the consequences as it is reduced. Finally we note that while smoothing $\kappa$ over a scale $\delta$ is crucial as it limits the scale of the formation of steps, capping the value of $\kappa$ is less important. A number of ways of doing this (including not doing so) were explored, and gave results that are very similar: the choice only changes just how flat the vorticity steps are, not their formation nor their merger and the stripping at the vortex edge.

\section{Random strain in the model of Balmforth et al. (2001)}

\subsection{Analytical development}

For the Gaussian vortex we observed a clear range of radii where the effective diffusivity is enhanced, and where steps in the profile emerge and merge. The core of the vortex (for example $0 \leqslant r \leqslant 2$ ) is relatively unscathed. This suggests exploiting an asymptotic framework in which a simplified model is used for the vortex core. Fortunately such a framework exists and is derived in Balmforth et al. (2001). The starting point is a compact vortex, for example a Rankine vortex, with vorticity strictly zero at some radius, the edge of the vortex. The vortex is assumed to support a neutral, normal mode whose critical layer, where fluid particles corotate with the mode, lies at a radius $r_{\mathrm{c}}$ which is outside the vortex. The model consists of an ordinary differential equation (ODE) for the complex amplitude $\hat{\varphi}(t)$ of the normal mode and a partial differential equation (PDE) for the evolution of weak vorticity $\zeta$ in the thin critical layer about the radius $r_{\mathrm{c}}$. This model captures at a qualitative level many phenomena that can occur in a Gaussian vortex subject to external strain fields: the ODE models the $\mathrm{e}^{ \pm \mathrm{i} m \theta}$ wave-like distortions of the central core, while the PDE captures the formation of cat's eyes and mixing of vorticity on the periphery. The two are coupled: the normal mode creates mixing in the layer, and mixing of vorticity can feed back to stabilize or destabilize the normal mode.

The governing equations for the forced model are

$$
\begin{gathered}
\partial_{t} \zeta+y \partial_{\theta} \zeta+\left[\partial_{y}(y+\zeta)\right] \partial_{\theta} \varphi=0, \\
\mathrm{i} \partial_{t} \hat{\varphi}=\varepsilon q(t)+\mathscr{P} \int_{-\infty}^{\infty} \mathrm{d} y \oint \frac{\mathrm{d} \theta}{2 \pi} \zeta \mathrm{e}^{-\mathrm{i} m \theta}, \\
\varphi(\theta, t)=\hat{\varphi}(t) \mathrm{e}^{\mathrm{i} m \theta}+\text { c. c. }
\end{gathered}
$$

We refer the reader to Balmforth et al. (2001) for a derivation and detailed description and only note here that in (3.1) $y$ is a scaled inwardly pointing radial coordinate with $y=0$ corresponding to the centre of the critical layer. The vorticity in the layer is $y+\zeta(y, \theta, t)$, and is transported in the angular velocity of the vortex, the $y \partial_{\theta} \zeta$ term, and because of radial motion from the normal mode via the term involving the flow $\partial_{\theta} \varphi$. This flow is simply linked by (3.3) to the amplitude $\hat{\varphi}(t)$ of the normal mode, governed by the ODE (3.2). In this equation the weak external random flow $\varepsilon q(t)$ is present, plus a feedback from the vorticity in the critical layer involving a principal value integral over $y$.

We exploit the limit $\varepsilon \rightarrow 0$ by introducing a long time scale $\tau=\varepsilon^{2} t$, and a weakly nonlinear expansion

$$
\zeta(y, t, \tau)=\bar{\zeta}(y, \tau)+\varepsilon \hat{\zeta}(y, t, \tau) \mathrm{e}^{\mathrm{i} m \theta}+\text { c. c. }+\cdots .
$$


Replacing $\hat{\varphi}$ by $\varepsilon \hat{\varphi}$, on the fast $t$ time scale we have the system

$$
\begin{gathered}
\partial_{t} \hat{\zeta}+\mathrm{i} m y \hat{\zeta}+\left(1+\partial_{y} \bar{\zeta}\right) \mathrm{i} m \hat{\varphi}=0, \\
\mathrm{i} \partial_{t} \hat{\varphi}=q+\mathscr{P} \int_{-\infty}^{\infty} \mathrm{d} y \hat{\zeta} .
\end{gathered}
$$

This gives advection of vorticity fluctuations on a mean, background distribution $y+\bar{\zeta}$ that depends on $y$ but does not vary on the short time scale $t$. On the longer $\tau$ time scale we have a conservation equation for the mean profile

$$
\partial_{\tau} \bar{\zeta}+\partial_{y} F=0, \quad F=2 m \operatorname{Im}\left\langle\hat{\zeta} \hat{\varphi}^{*}\right\rangle
$$

Here the brackets denote both an average over $t$ as well as an ensemble average over the distribution of random flow amplitudes $q(t)$. From now on we drop the hats from fluctuating quantities; we shall also not stress the dependence on $\tau$ of the various quantities in the problem.

Our plan is to solve the fluctuating problem (3.5) and (3.6) for $\zeta$ and $\varphi$ with any background mean profile $\bar{\zeta}$, and then isolate the flux $F$ in (3.7) to give a diffusion equation for this profile on the long time scale. Using (2.11), (3.5) and (3.6) in Laplace transform space become

$$
\begin{gathered}
(-\mathrm{i} p+\mathrm{i} m y) \tilde{\zeta}+\mathrm{i} m\left(1+\partial_{y} \bar{\zeta}\right) \tilde{\varphi}=0, \\
p \tilde{\varphi}=\widetilde{q}+\mathscr{P} \int_{-\infty}^{\infty} \mathrm{d} y \widetilde{\zeta},
\end{gathered}
$$

and are solved straightforwardly (with zero initial conditions) so that

$$
\widetilde{\zeta}(p)=K(y, p) M(p) \widetilde{q}(p), \quad \tilde{\varphi}(p)=M(p) \widetilde{q}(p),
$$

where

$$
K(y, p)=\frac{1+\partial_{y} \bar{\zeta}(y)}{p / m-y}, \quad L(p)=\mathscr{P} \int_{-\infty}^{\infty} \mathrm{d} y K(y, p), \quad M(p)=\frac{1}{p-L(p)} .
$$

Here the principal value integral for $L(p)$ refers to the limit $y \rightarrow \pm \infty$, but in addition we need to note that the contour $\Gamma$ sits above the real axis, and when brought down meets a pole at $p=m y$ from the definition of $K(y, p)$. We can extract the integral over the singular component $\left(1+\partial_{y} \bar{\zeta}(p / m)\right) /(p / m-y)$ and evaluate this exactly for $p$ above the real axis. There is then no obstruction to bringing the contour $\Gamma$ down to the real axis, in other words making $p$ real. Writing $L=L_{\mathrm{r}}+\mathrm{i} L_{\mathrm{i}}$ in terms of real and imaginary parts we have

$$
L_{\mathrm{r}}(p)=-\mathscr{P} \int_{-\infty}^{\infty} \mathrm{d} y \frac{\partial_{y} \bar{\zeta}(y)-\partial_{y} \bar{\zeta}(p / m)}{y-p / m}, \quad L_{\mathrm{i}}(p)=-\pi\left(1+\partial_{y} \bar{\zeta}(p / m)\right) .
$$

This solution may seem rather formal but given a profile $\bar{\zeta}(y), L$ and $M$ are easily obtained numerically for any real value of $p$. Note that if the vorticity gradient is a constant, $\bar{\zeta}(y)=0$, then $L(p)=-\mathrm{i} \pi$. This gives a simple pole in

$$
M(p)=(p+\mathrm{i} \pi)^{-1}
$$

at $p=-\mathrm{i} \pi$ which is used in the formula (3.16) below. This pole corresponds to the quasi-mode damping rate $\mathrm{e}^{-\mathrm{i} p t}=\mathrm{e}^{-\pi t}$ (as in Balmforth et al. 2001). For more general profiles a computation of $M(p)$ will determine the damping rate for quasi-modes (e.g. Hall, Bassom \& Gilbert 2003). 
Given this solution it is now just a question of computing the average $\left\langle\hat{\zeta} \hat{\varphi}^{*}\right\rangle$ that provides the flux $F$ in (3.7). This calculation is described in Appendix A: the result is that the vorticity $\bar{\omega}=y+\bar{\zeta}(y, \tau)$ in the layer is governed by the diffusion equation for $\bar{\zeta}$

$$
\partial_{\tau} \bar{\zeta}+\partial_{y} F=0, \quad F(y)=-\kappa(y) \partial_{y}(y+\bar{\zeta}),
$$

with diffusivity

$$
\kappa_{*}(y)=m^{2}|M(p)|^{2} w(p), \quad p \equiv m y .
$$

Here we have the weight function $w(p)$ from (2.5) or (2.6) and the quantity $M(p)$ which gives the response of the vortex to frequency $p$ and is determined by an integration over the vorticity distribution in the layer (see (3.11)): as a result the diffusivity depends nonlinearly and non-locally on the profile $y+\bar{\zeta}(y)$. At $\tau=0$ with a uniform gradient initially, $\bar{\omega}=y$, we have

$$
\kappa_{*}(y)=m^{2}\left(m^{2} y^{2}+\pi^{2}\right)^{-1} w(p),
$$

and this is shown in figure $1(b)$ (for $w(p) \equiv 1$ ). This is analogous to the peak in the curve in figure 1(a), confirming that the framework in Balmforth et al. (2001) should model the transport in the tail of the Gaussian vortex. As in the Gaussian case, we cap and smooth $\kappa_{*}$ using a parameter $\delta$ to obtain $\kappa$, with

$$
\kappa=G_{\delta} F_{\delta}\left(\kappa_{*}\right) .
$$

We again have in mind linking $\delta$ to the quantity $\varepsilon^{1 / 2}$, where $\varepsilon \ll 1$ is the strength of the forcing. (Note that in this case we have not normalized by a scalar diffusivity as there is no power law growth with increasing $y$.)

\subsection{Numerical results}

Figures 4 and 5 show numerical results for simulations of the diffusion equation (3.14) and (3.15) with $M(p)$ computed as detailed in (3.11) and (3.12). Figure $4(a, b)$ shows vorticity profiles analogous to those of $\bar{\omega}$ in the left panels of figure 2 , bearing in mind that $y$ increases radially inwards. The corresponding effective diffusivities are shown in the right panels of the two figures. There are strong similarities between the full evolution of the Gaussian initial condition and the simplified model of Balmforth et al. (2001), confirming the usefulness of the model in capturing many phenomena at a qualitative level. The model system shows the development of a vorticity staircase. The flattened regions are characterized by strongly enhanced diffusivity: in between there are transport barriers, where the reduced diffusivity allows high gradients to persist.

The butterfly diagrams in figure 5 are analogous to those in figure 3 for the Gaussian case. Again we see many similarities: steps are formed and merge, and there are also ripples at the edge, moving towards the origin $y=0$, particularly visible in $5(c, d)$, and seen in the Gaussian case in figure 3. Of course there are also differences, and we stress that the model of Balmforth et al. (2001) is not an asymptotic approximation to a Gaussian vortex so comparisons are only qualitative. The key difference appears to be that in the model the critical layer is embedded in an infinitely wide vorticity gradient, on the appropriate small scale $y$, as is clear in figure 4 . The gradient traps the vorticity staircase, while the effective diffusivity $\kappa(y)$ drops away on either side (figure $1 b$ ). This matches the behaviour for the Gaussian vortex around $r=4$, but there the random strain field takes over for increasing radii and the effective diffusivity increases, as seen in figure $1(a)$. This tends to make the Gaussian picture asymmetrical, and allows vorticity to be stripped to infinity, not possible in the model. 
(a)

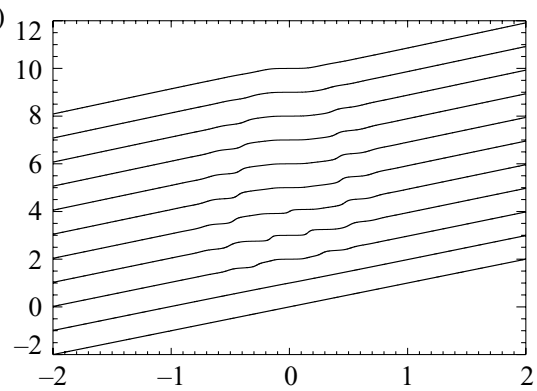

(b)

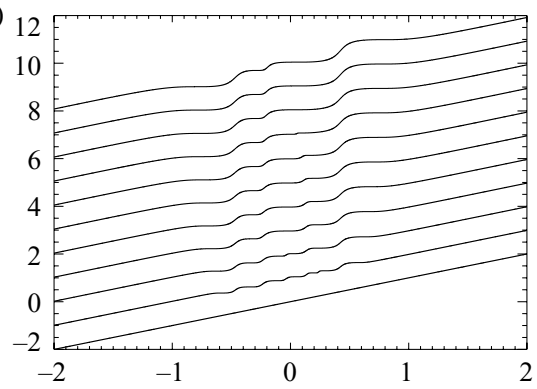

(c)

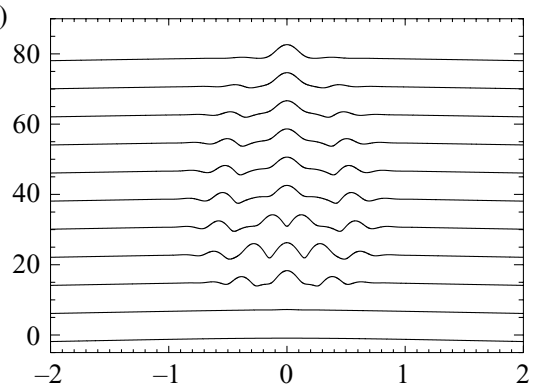

(d)

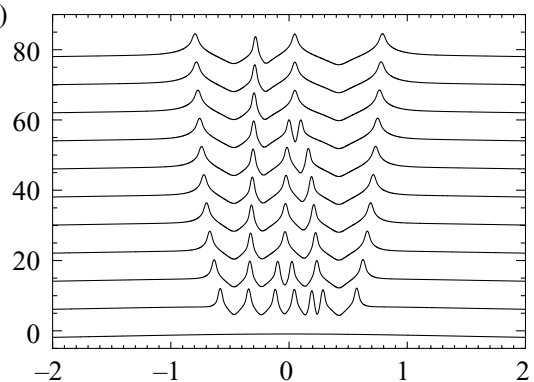

Figure 4. Evolution in the model of Balmforth et al. (2001) with smoothing $(a, c) \delta=0.05$ and $(b, d) \delta=0.01$. The curves show a sequence of vorticity profiles $\bar{\omega}=y+\bar{\zeta}(a, b)$ and effective diffusivities $\log \kappa(c, d)$, plotted against $y$. In each panel the curves are separated by additive constants and given for $\tau=0,0.1,0.2$, etc., reading up the curves.

(Note that there is some symmetry breaking in the numerical runs in figure $5(d, e)$ : the mathematical problem is strictly symmetric in $y$, but instabilities soon break this, whether seeded by rounding or truncation error, or by initializing $\bar{\zeta}$ with weak noise, as we do.)

We observe from figure 5 that as the smoothing parameter $\delta$ is reduced we obtain finer scales in the problem, and a more rapid onset of the growth of steps, over a very clearly defined range of $y$ values. To see this clearly figure 6 shows a run with $\delta=0.005$, the smallest value used, with evolution over a short time interval in figure $6(a)$ and over a longer interval in figure $6(b)$. Over the short time we see a rapid growth of fine scale structure and waves (limited by the value of $\delta$ ); these form finely spaced steps which then show a merger process, over both short times in figure $6(a)$ and longer times in figure $6(b)$. The initial development at first suggests a 'negative diffusion' type instability with a growth rate increasing with wavenumber $k$ of the initial fluctuations, perhaps as $O\left(k^{2}\right)$. For example, this is reminiscent of the Cahn-Hilliard equation where diffusion with a negative diffusivity is controlled on the smallest scales by a fourth derivative: fine structure develops initially and then scales increase as phase separation occurs and domains merge. However in our simulations $\kappa(y) \geqslant 0$ at all times, and also $\kappa(r) \geqslant 0$ in the Gaussian case. The initial development of fine structure arises through the term $\left(\partial_{y} \kappa\right) \partial_{y}(y+\bar{\zeta})$, from expanding the diffusive term $\partial_{y}\left(\kappa \partial_{y}(y+\bar{\zeta})\right)$ in $(3.14)$ as we now explain.

Suppose we start a linear vorticity profile $\bar{\omega}=y$, and add on a weak fine scale vorticity distribution $\bar{\zeta}(y)=\mu \sin k y$ with $\mu \ll 1$ and $k \gg 1$. Now the corresponding $\kappa$ will also be perturbed from (3.16) with a component of magnitude $\mu$ and wavenumber $k$. By calculating this it can be shown that at a point $y$ the original disturbance will 
(a)

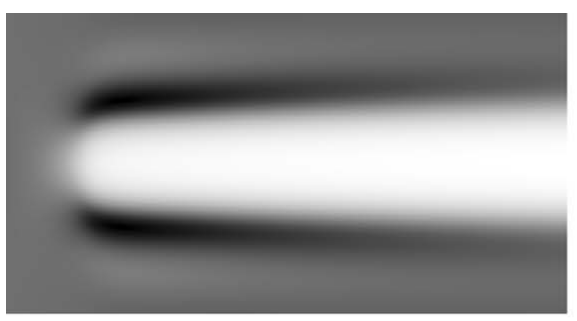

(b)

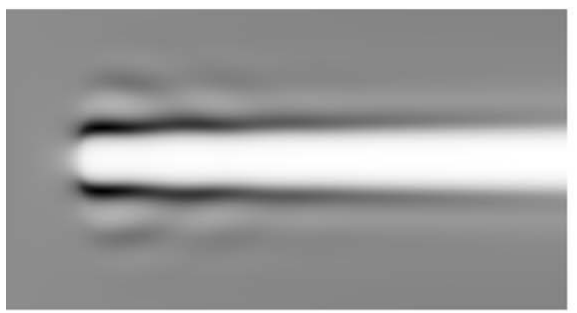

(c)

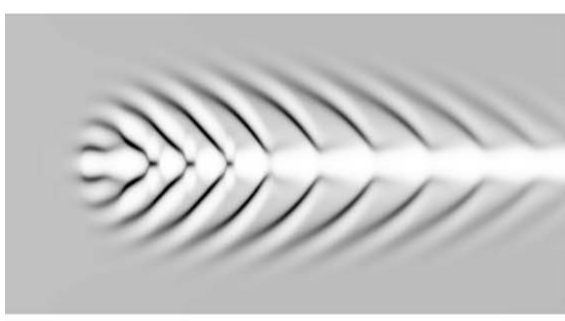

(d)

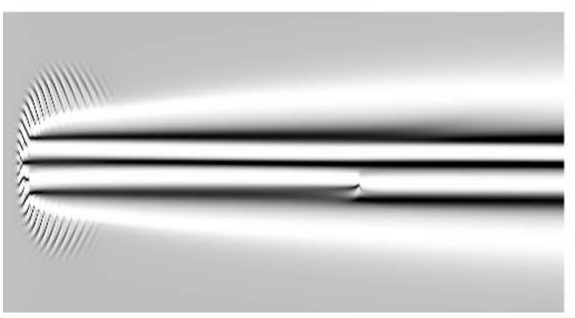

(e)

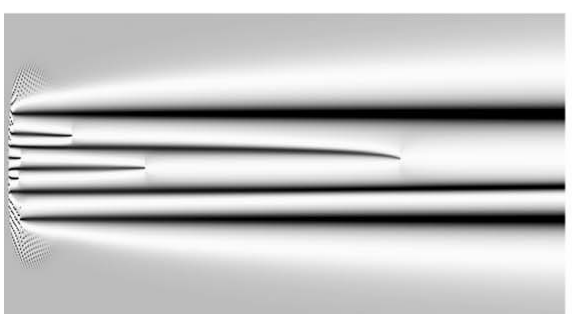

(f)

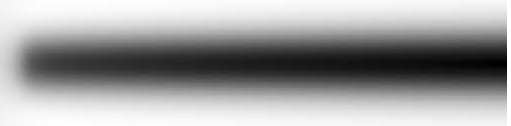

(g)

(h)



(i)



(j)



FIGURE 5. Space-time diagram of the evolution of the perturbation vorticity gradient $\partial_{y} \bar{\zeta}(y, \tau)$ $(a-e)$ and the effective diffusivity $\kappa(y, \tau)(f-j)$, plotted in grey scales in the $(\tau, y)$ plane for $(a, f)$ $\delta=0.2,(b, g) \delta=0.1,(c, h) \delta=0.05,(d, i) \delta=0.02$ and $(e, j) \delta=0.01$. The grey scale coding is capped at the level of 4 , corresponding to black; zero is white. The ranges are $-2 \leqslant y \leqslant 2$ (vertical) and in the horizontal, $(a, f) 0 \leqslant \tau \leqslant 4$ and $(b, g) 0 \leqslant \tau \leqslant 2$ and $(c-e, h-j) 0 \leqslant \tau \leqslant 1$. 
(a)

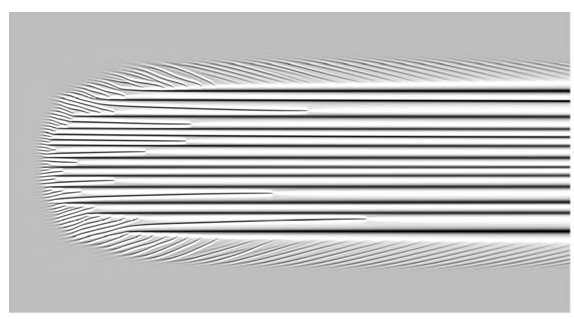

(b)

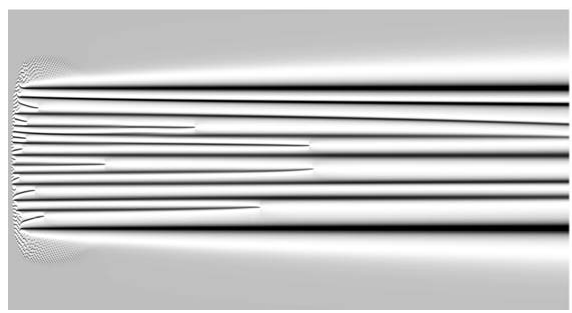

(c)

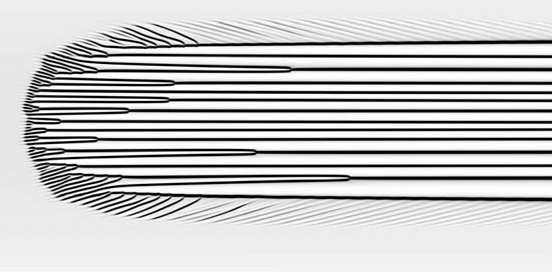

(d)

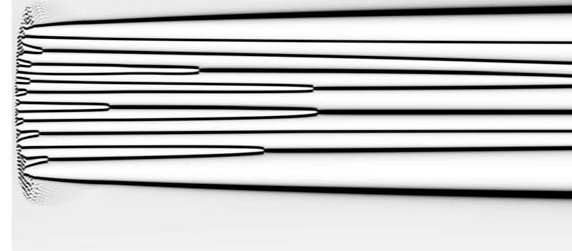

Figure 6. As in figure 5 for $\delta=0.005$ and $(a, c)$ short times $0 \leqslant \tau \leqslant 0.025$ and $(b, d)$ moderate times $0 \leqslant \tau \leqslant 0.25$.

have a local growth rate of

$$
\gamma(y) \simeq k^{2} m^{2}\left(m^{2} y^{2}+\pi^{2}\right)^{-2} w(m y)\left(\pi^{2}-m^{2} y^{2}\right),
$$

and so the disturbance grows with a growth rate proportional to $k^{2}$ (similar to a 'negative diffusivity' instability but without $\kappa$ changing sign) in the band $|y| \leqslant \pi / m$. The range of $y$ for which modes are predicted to grow is consistent with the emergence of fine scales in figures 5 and 6 ; for example the latter figure shows an absence of fine scales outside the range $|y| \lesssim 1.4$, to be compared with the prediction $|y|<\pi / 2 \simeq$ 1.57. This band is perhaps a little narrower than the range indicated from theory, presumably because of nonlinear effects that will occur most quickly at the centre, but have a wider influence.

This calculation is detailed in Appendix C. We believe this also gives an interpretation for the formation of ever finer scales in the Gaussian vortex as $\delta \rightarrow 0$, and think it is likely that there is a range of radii at which this can occur. It would be interesting to determine the range, but we do not know how to do this.

\section{Discussion}

We have investigated the evolution of a coherent two-dimensional vortex subjected to weak external random strain, both starting with a Gaussian vortex and using the asymptotic model (Balmforth et al. 2001) of compact vortex core and weak vorticity skirt. Averaging over the external random strain allowed us to write down a diffusion equation for the mean vorticity profile. The effective diffusivity is linked explicitly to the stability properties of the profile: although the link is nonlinear and non-local, it can be calculated to give the diffusivity as a function of radius for any given vorticity profile.

We then time-stepped this diffusion equation to attempt to understand the coupling between mixing of vorticity and the vortex response for axisymmetric flow starting with a Gaussian vortex and for the model of Balmforth et al. (2001). We have found that the dynamical response gives similar features in both cases with the generation of fine scale steps forming a vorticity staircase. In the flat regions of the steps the effective diffusivity is large, while in between small values of the effective diffusivity allow high 



FIGURE 7. Schematic of process of quasi-mode damping suppressing mixing in the vertical part of a vorticity step, as described in the text.

gradients of vorticity to persist and form transport barriers. Merger processes tend to reduce the number of steps, and in the Gaussian case we find that vorticity is stripped to infinity, leaving a sharp-edged vortex. Surrounding this is a region where the effective diffusivity is large.

Much of what we see in the Gaussian case is reproduced in the model of Balmforth et al. (2001), which allowed us both to pursue higher numerical resolutions, and to understand the initial development of fine scales. In addition we can give a heuristic description of how the sharp vertical parts of steps are maintained in the profile, shown in figure 7 (see Hall et al. 2003). It amounts to the fact that there is damping of quasi-modes (corresponding to a normal mode on the vortex core) in the presence of a vorticity gradient at the appropriate critical radius. In figure $7(a)$ there is external rotating strain, frequency $p$, acting on a vortex core surrounded by a corotating vertical vorticity step at $r$ with $p=m \alpha(r)$. The external strain distorts the step into an ellipse in $(b)$, creating the $+/-$ vorticity anomaly and flow shown. This distorts the vortex core giving the secondary vorticity anomaly and flow indicated in $(c)$, which has the effect of countering the original external strain and restoring the step to circular form. Thus an external frequency that is tuned to mix vorticity in the vertical part of the step is damped and mixing suppressed. For a frequency that corresponds to a flat part of a step and a low gradient at the critical radius, no vorticity anomaly is created and so there is no damping mechanism.

The development of vorticity staircases and inhomogeneous mixing is mirrored in observations and simulations of geophysical flows. For example, simulations of Norton (1994) and Waugh \& Plumb (1994) of stratospheric polar vortex dynamics indicate a coherent vortex core, undergoing significant distortions (greater than in our study), surrounded by a 'surf zone' of breaking Rossby waves (McIntyre \& Palmer 1984). Such sharp-edged vortices can also be generated by the process of vortex stripping (e.g. Mariotti, Legras \& Dritschel 1994), where the flow generates a hyperbolic separatrix at the edge of the vortex, which erodes vorticity on a very rapid time scale. The stripping we see, which leaves a sharp edge in simulations of the Gaussian vortex, is in some ways a milder process, as it results from the accumulation of weak random external strain over a long time scale: nonetheless it too results from the algebraic growth of the random strain field with radius. Steps in potential vorticity fields are also seen in atmospheric data; see for example Dritschel \& McIntyre (2008), who review observations, discuss physical mechanisms and present numerical simulations. Here the vorticity gradients in our models are replaced by a background vorticity gradient or $\beta$-effect, which governs jet width scaling in geophysical problems. Banded 
structures are seen in giant gaseous planets, most notably Jupiter and Saturn, and emerge in simulations of randomly forced spherical shallow water models and in convective deep models (Rotvig \& Jones 2006; Heimpel \& Aurnou 2007; Scott \& Polvani 2007). It would be interesting to extend the present study to derive a diffusion equation for fluid motions on a sphere in the case of a two-dimensional fluid or a shallow water system, building on the weakly nonlinear study of Garaud (2001), who followed the effects of shear instability in flows relevant to the solar tachocline.

The weakest part of our study is the necessity to introduce a smoothing (and capping) of the effective diffusivity, via the parameter $\delta$. This is forced upon us by the presence of instabilities of arbitrarily rapid growth rate, on the long time scale. These would in practice be controlled by nonlinearity on the short time scale, which is not present in our analysis, nor are we aware of any easy way in which to incorporate it. The next step is to see whether full simulations of randomly forced vortices, with nonlinearity, reproduce the results seen here and to see how far our assumptions of weak external forcing, and consequent evolution of the profile on a long time scale, may be relaxed. Also, because we cannot justify the form of the smoothing and capping parameter $\delta$, we have not explored the very long time limit $\tau \rightarrow \infty$ : we expect mergers of steps in the two models to occur, over increasingly lengthy time scales, leaving a step-less compact vortex in the Gaussian case. This would be interesting to explore in a full numerical model.

A further direction of research would be in understanding the nature and role of the external forcing $q(t)$ and its weight function $w(p)$ in more realistic flows. For example, in two-dimensional turbulence the irrotational flows of magnitude $\varepsilon$ generated by other vortices would vary on a slow time scale of order $\varepsilon t$, corresponding to a peak of $w(p)$ at the corresponding low frequencies. The effects we have discussed would then come from the high-frequency tail of this peak: although $w(p)$ would fall off, the dynamics of the vortex tends to amplify the effects of frequencies $p$ which interact with the quasi-mode, i.e. via the resonant peak seen in figure $1(a)$, and pick out the frequencies that give mixing and step formation at the periphery of the vortex on the slower $\varepsilon^{2} t=\tau$ time scale. This would again be best studied by means of numerical simulations.

We thank the referees for helpful comments which improved the paper. We are grateful to the Leverhulme Trust which supported A. D. Gilbert by a Leverhulme Research Fellowship during 2007-2008, and to the EPSRC for research grant EP/D032202/1, which supported M. R. Turner and annual visits of A. P. Bassom to Exeter. We are pleased to acknowledge useful discussions and references from David Dritschel, Stéphane Le Dizès and John Thuburn.

\section{Appendix A. Feedback on the mean profiles}

Here we discuss the feedback from the fluctuations on the mean profiles, firstly in the case in Balmforth et al. (2001), and then in the axisymmetric (initially Gaussian) case. These require the calculation of $F$ in (3.7) and (2.10), respectively. To handle complex conjugation in the Laplace transform framework we proceed as follows; note that we always keep the coordinates $r$ or $y$ and time $t$ real in our analysis. Given a complex analytic function $f(z)$ we define another one, $f^{*}(z)$, by

$$
f^{*}(z)=\left(f\left(z^{*}\right)\right)^{*}
$$

whereupon we have

$$
\widetilde{f}^{*}(p)=\int_{0}^{\infty} \mathrm{d} t \mathrm{e}^{-\mathrm{i} p t} f^{*}(t), \quad f^{*}(t)=-\frac{1}{2 \pi} \int_{\Gamma^{\prime}} \mathrm{d} p \mathrm{e}^{\mathrm{i} p t} \widetilde{f}^{*}(p),
$$


where $\Gamma^{\prime}$ goes from $+\infty$ to $-\infty$ below all the singularities of $\widetilde{f}^{*}(p)$ and so is at the outset the mirror image in the real axis of the contour $\Gamma$.

For the random amplitude $q(t)$ of the forcing, we will need

$$
\left\langle\widetilde{q}(p) \widetilde{q}^{*}\left(p^{\prime}\right)\right\rangle=\int_{0}^{\infty} \mathrm{d} t \int_{0}^{\infty} \mathrm{d} t^{\prime}\left\langle q(t) q^{*}\left(t^{\prime}\right)\right\rangle \mathrm{e}^{\mathrm{i}\left(p t-p^{\prime} t^{\prime}\right)},
$$

which in the delta correlated case $(2.5)$ is

$$
\left\langle\widetilde{q}(p) \widetilde{q}^{*}\left(p^{\prime}\right)\right\rangle=\mathrm{i}\left(p-p^{\prime}\right)^{-1},
$$

and in the exponential case (2.6) is

$$
\left\langle\widetilde{q}(p) \widetilde{q}^{*}\left(p^{\prime}\right)\right\rangle=\frac{a^{2}}{\left(p^{\prime}-m b\right)^{2}+a^{2}} \frac{\mathrm{i}}{p-p^{\prime}}+\frac{a}{2\left(p^{\prime}-m b+\mathrm{i} a\right)(p-m b+\mathrm{i} a)} .
$$

A.1. The model of Balmforth et al. (2001)

The feedback onto the mean profile in (3.7) is governed by $\left\langle\zeta(y, t) \varphi^{*}(t)\right\rangle$ and we have

$$
(-2 \pi)^{2} \zeta(y, t) \varphi^{*}(t)=\left(1+\partial_{y} \bar{\zeta}\right) \int_{\Gamma} \mathrm{d} p \int_{\Gamma^{\prime}} \mathrm{d} p^{\prime} \frac{\mathrm{e}^{-\mathrm{i}\left(p-p^{\prime}\right) t}}{p / m-y} M(p) M^{*}\left(p^{\prime}\right) \widetilde{q}(p) \widetilde{q}^{*}\left(p^{\prime}\right),
$$

by (2.11), (A 2), (3.10) and (3.11). For our purposes we need to assume that $M(p)$ is analytic except for singularities $S$ in a set bounded below the real axis. The distance $\Delta=\Delta(\tau)$ of $S$ below the real axis, i.e. the width of the analyticity strip of $M(p)$, will generally decrease with $\tau$ as the vorticity profile evolves, and the theory breaks down if $\Delta(\tau)$ becomes zero: this is in fact what happens in the simulations, and why we have to adopt a smoothing for the resulting large values of the diffusivity, modelling nonlinear saturation of the mixing. Although we have to adopt this ad hoc procedure for the simulations, we set out the theory with the assumption that the profile at the given time $\tau$ is stable, i.e. $\Delta(\tau)>0$.

Ensemble averaging and substituting the correlation function (A 4) for the delta correlated case gives

$$
(-2 \pi)^{2}\left\langle\zeta(y, t) \varphi^{*}(t)\right\rangle=\left(1+\partial_{y} \bar{\zeta}\right) \int_{\Gamma} \mathrm{d} p \int_{\Gamma^{\prime}} \mathrm{d} p^{\prime} \frac{\mathrm{e}^{-\mathrm{i}\left(p-p^{\prime}\right) t}}{p / m-y} \frac{\mathrm{i}}{p-p^{\prime}} M(p) M^{*}\left(p^{\prime}\right) .
$$

Figure 8 depicts the contours $\Gamma$ and $\Gamma^{\prime}$ in the complex plane together with the singularities $S$ of $M(p)$ below the real axis, and singularities $S^{*}$ of $M^{*}\left(p^{\prime}\right)$ above. We close the $\Gamma^{\prime}$ contour in the upper half plane using the exponential decay of $\mathrm{e}^{\mathrm{i} p^{\prime} t}$ there. This encloses the pole $p^{\prime}=p$ (but no pole at $m y$ ) and any other singularities $S^{*}$ of $M^{*}\left(p^{\prime}\right)$. We can ignore the effect of $S^{*}$ as singularities contribute only exponentially decaying terms, decaying at least as $\mathrm{e}^{-\Delta(\tau) t}$, that disappear on the fast time scale. In other words we are implicitly averaging the right-hand side over the fast time scale, although we have not put in angled brackets for reasons of readability. We are left with

$$
(-2 \pi)\left\langle\zeta(y, t) \varphi^{*}(t)\right\rangle=\left(1+\partial_{y} \bar{\zeta}\right) \int_{\Gamma} \mathrm{d} p \frac{1}{p / m-y} M(p) M^{*}(p) .
$$

We note that we in fact only require the imaginary part to form the flux $F$ in (3.7). To deal with this integral, we bring the contour down to lie along the real axis, except at the pole $p=m y$. On the real axis, since

$$
M(p) M^{*}(p)=|M(p)|^{2} \text { for } p \text { real }
$$




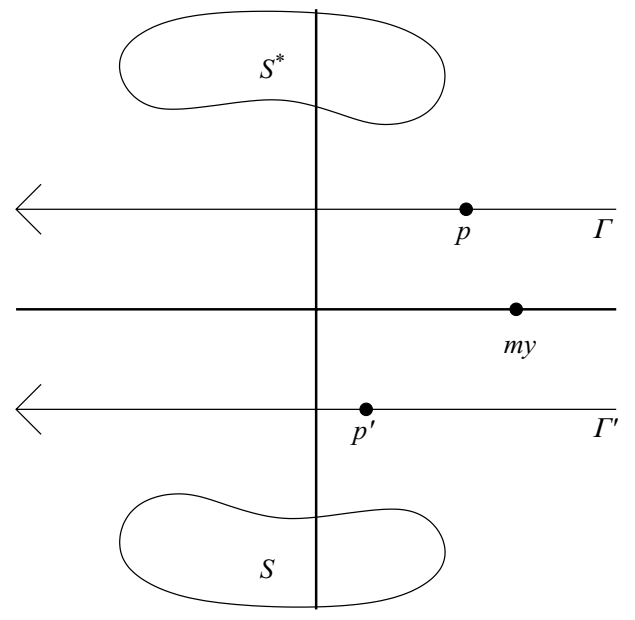

FIGURE 8. Schematic picture of contours $\Gamma$ and $\Gamma^{\prime}$ in the complex plane, discussed in the text.

from (A 1), we only obtain a real contribution to the integral, which we do not need. Integrating anticlockwise around the semicircle above the pole $p=m y$ leaves an imaginary contribution of $i \pi$ times the residue to give

$$
(-2 \pi) \operatorname{Im}\left\langle\zeta(y, t) \varphi^{*}(t)\right\rangle=\left(1+\partial_{y} \bar{\zeta}\right) \pi m|M(m y)|^{2} .
$$

This yields the flux and effective diffusivity

$$
F=-\kappa(y)\left(1+\partial_{y} \bar{\zeta}\right), \quad \kappa(y)=m^{2}|M(m y)|^{2} .
$$

The calculation is similar for the exponential correlation function (2.6) with (A 5) and introduces the factor $w(p)$ into (3.15).

We present an alternative derivation of the result for $\kappa(y)$ : we need this so that we can proceed more swiftly in the axisymmetric case next. We start with the complex conjugate version of (A 6), which is, after substituting the correlation function (A 4),

$$
(-2 \pi)^{2}\left\langle\varphi(y, t) \zeta^{*}(t)\right\rangle=\left(1+\partial_{y} \bar{\zeta}\right) \int_{\Gamma} \mathrm{d} p \int_{\Gamma^{\prime}} \mathrm{d} p^{\prime} \frac{\mathrm{e}^{-\mathrm{i}\left(p-p^{\prime}\right) t}}{p^{\prime} / m-y} \frac{\mathrm{i}}{p-p^{\prime}} M(p) M^{*}\left(p^{\prime}\right),
$$

and close the $p^{\prime}$ contour in the upper half plane. Now we pick up the residues from the poles at $p^{\prime}=p$ and at $p^{\prime}=m y$ in figure 8; the result can be written as

$$
(-2 \pi)\left\langle\varphi(y, t) \zeta^{*}(t)\right\rangle=\left(1+\partial_{y} \bar{\zeta}\right) \int_{\Gamma} \mathrm{d} p \frac{M(p) M^{*}(p)}{p / m-y}\left(1-\frac{M^{*}(m y)}{M^{*}(p)} \mathrm{e}^{-\mathrm{i}(p-m y) t}\right) .
$$

This integral has a removable singularity at $p=m y$ and the integration contour may be moved to lie entirely along the real axis! However we remember that we only require the imaginary part of this expression and that we are implicitly averaging over time. Except within an $O\left(t^{-1}\right)$ neighbourhood of $p=m y$ the exponential averages to zero to leave a quantity that is purely real and so can be discarded. The imaginary part of the integral comes only from this neighbourhood and is at leading order

$$
(-2 \pi) \operatorname{Im}\left\langle\varphi(y, t) \zeta^{*}(t)\right\rangle=-\left(1+\partial_{y} \bar{\zeta}\right)|M(m y)|^{2} \int_{m y-T}^{m y+T} \mathrm{~d} p \frac{\sin ((p-m y) t)}{p / m-y},
$$

where we have set $t^{-1}<T \ll 1$ and reversed the contour direction. The integral here is $m \pi$ at leading order, and so the result is in agreement with (A 10). 


\section{A.2. Axisymmetric flow}

We now turn to the axisymmetric case in which the feedback on the mean profile in (2.10) involves

$$
(-2 \pi)^{2}\left\langle\psi(r, t) \omega^{*}(r, t)\right\rangle=\int_{\Gamma} \mathrm{d} p \int_{\Gamma^{\prime}} \mathrm{d} p^{\prime} M(r, p) N^{*}\left(r, p^{\prime}\right) \frac{\mathrm{i}}{p-p^{\prime}} \mathrm{e}^{-\mathrm{i}\left(p-p^{\prime}\right) t},
$$

for the delta correlated case. For each value of $p$ we close the $p^{\prime}$ contour. The situation is depicted in figure 8, except that $m y$ is replaced by $m \alpha(r)$. From (2.15) with $p^{\prime}=m \alpha\left(s^{\prime}\right)$ replacing $p$ there is a pole singularity in $N\left(r, p^{\prime}\right)$ as now $p^{\prime}$ varies, which takes the form

$$
N\left(r, p^{\prime}\right)=-C(m \alpha(r)) m \beta(r)\left(m \alpha(r)-p^{\prime}\right)^{-1}+\cdots
$$

(using the definition of $\mu(r)$ below (2.17)). After picking up the residues from the two poles $p^{\prime}=p$ and $p^{\prime}=m \alpha(r)$ of the $p^{\prime}$ integration we are left with

$$
(-2 \pi)\left\langle\psi(r, t) \omega^{*}(r, t)\right\rangle=\int_{\Gamma} \mathrm{d} p M(r, p)\left(N^{*}(r, p)-\frac{m \beta(r) C^{*}(m \alpha(r))}{p-m \alpha(r)} \mathrm{e}^{-\mathrm{i}(p-m \alpha(r)) t}\right) .
$$

The argument then follows that for the model of Balmforth et al. (2001) after (A 13). The singularity at $p=m \alpha(r)$ is removable and so we can deform the $\Gamma$ contour to the real axis and reverse its direction. Away from $p=m \alpha(r)$ the exponential averages to zero, and the product $M(r, p) N^{*}(r, p)$ is real (as $\widetilde{\psi}$ is a real multiple of $\widetilde{\omega}$ in (2.12)). In the $O\left(t^{-1}\right)$ neighbourhood of $p=m \alpha(r)$ only, there is a contribution to the imaginary part, leaving

$$
2 \pi \operatorname{Im}\left\langle\psi(r, t) \omega^{*}(r, t)\right\rangle=\pi m \beta(r)|C(m \alpha(r))|^{2} .
$$

The resulting flux can be written as

$$
F=-\kappa(r) \partial_{r} \bar{\omega}, \quad \kappa(r)=m^{2} r^{-2}|C(m \alpha(r))|^{2} .
$$

Similarly, for advection of a passive scalar we instead solve

$$
\partial_{t} \sigma+\mathrm{i} m \alpha \sigma=-\mathrm{i} m \beta \psi=-\mathrm{i} m \beta r^{m} q(t),
$$

where $\alpha(r)$ is the angular velocity of a given flow field and $\beta=r^{-1} \partial_{r} \bar{\sigma}$ to obtain (2.20).

\section{Appendix B. Numerical methods}

The diffusion equation for the system in Balmforth et al. (2001)

$$
\partial_{\tau} \bar{\zeta}=\partial_{y}\left(\kappa(y) \partial_{y} \bar{\zeta}\right)
$$

with (3.11) and (3.15), was simulated numerically using a simply constructed code, second order in space and time, in which the variable $y$ is discretized with $N$ points $y_{j}$ in the interval $[-Y, Y]$. We use a corresponding grid of points $p_{j}=m y_{j}$ in the interval $[-m Y, m Y]$ where $Y$ is chosen to be large enough to capture all the interesting behaviour in $\bar{\zeta}$. We set up the initial conditions and add very weak random noise to the vorticity values (at a level of $10^{-6}$ ) to trigger instabilities (otherwise these are generated by rounding or truncation error and so dependent on machine precision or choice of grid and time step). The process is then as follows. 
We evaluate $\partial_{y} \bar{\zeta}$ at points $y_{j}$ on the grid using finite differences and then $L(p)$ at the grid points $p_{j}$ using (3.12) with $L_{r}(p)$ written as

$$
L_{\mathrm{r}}(p)=-\int_{-Y}^{Y} \mathrm{~d} y \frac{\partial_{y} \bar{\zeta}(y)-\partial_{y} \bar{\zeta}(p / m)}{y-p / m}+\partial_{y} \bar{\zeta}(p / m) \log \left|\frac{Y+p / m}{Y-p / m}\right| .
$$

From this we compute $\kappa_{*}$ at the gridpoints $y_{j}$ from (3.15) and (3.11). We cap and smooth $\kappa_{*}$ to give $\kappa$ according to (3.17).

The mean profile is then stepped in time using a modified Crank-Nicolson method. Starting with the current field at time $t=k \Delta t$, this method would usually involve solving nonlinear equations for the future field $\bar{\zeta}$ and the diffusivity $\kappa$ at time $t+\Delta t$. However as the diffusivity is a complicated functional of the field this is not practical. Thus the method is combined with a predictor-corrector step: the present diffusivity is held constant while the field alone is stepped from $t$ to $t+\Delta t$. This gives an estimate of the future field, from which an estimate of the future diffusivity may be computed and finally used to take another Crank-Nicolson step for the field from $t$ to $t+\Delta t$. This process can be iterated, but tests indicate that two steps (one to estimate the diffusivity and one to step the field) are sufficient. In figure 5, parameter values used are, in figure $5(a)(N, Y, \Delta t)=\left(8000,10,5 \times 10^{-4}\right)$, figure $5(b)$ $(N, Y, \Delta t)=\left(16000,10,2 \times 10^{-4}\right)$ and figure $5(c, d, e)(N, Y, \Delta t)=\left(32000,10,10^{-4}\right)$ and for figure $6,(N, Y, \Delta t)=\left(32000,6,2.5 \times 10^{-5}\right)$.

For the full, Gaussian problem, a similarly structured code was written to time step the diffusion equation

$$
\partial_{\tau} \bar{\omega}=r^{-1} \partial_{r}\left(r \kappa(r) \partial_{r} \bar{\omega}\right)
$$

with $\kappa$ given in (2.19) and $C(p)$ in (2.12)-(2.17). The code starts with $\bar{\omega}$ known on a radial grid $r_{j}$ with $N$ points from 0 to $R$. The quantities $p_{j}=\alpha\left(r_{j}\right)$ and $\beta\left(r_{j}\right)$ are calculated using a cubic spline fit while for each grid point $r_{j}$ the code computes $C\left(p_{j}\right)$ as follows. We let $h$ be a small positive numerical parameter and interpolate the relevant functions onto a finer grid with $M$ points, $M \gg N$. The ODE (2.12) is integrated from $r=h$ using $\widetilde{\psi}(h, p)=h^{m}$ and $\partial_{r} \widetilde{\psi}(h, p)=m h^{m-1}$ to $r=r_{j}-h$ using a fourth order Runge-Kutta scheme. The solution is then stepped over the critical point, from $r_{j}-h$ to $r_{j}+h$, using the jump conditions from (2.16), (2.17), namely,

$$
[\widetilde{\psi}]_{-}^{+}=0, \quad\left[\partial_{r} \widetilde{\psi}\right]_{-}^{+}=\mathrm{i} \chi \mu\left(r_{j}\right) \widetilde{\psi}\left(r_{j}\right)
$$

(in our case $\chi=-\pi$ ) and finally integrated out to $R$ where $\widetilde{\psi} \propto r^{m}$. The solution is then divided throughout by the (complex) constant of proportionality so as to satisfy (2.13) and the resulting value of $\widetilde{\psi}$ at $r=r_{j}$ is then just $C\left(p_{j}\right)$. Note that a check on this part of the code exists: solutions obtained for a Gaussian mean profile are given in figure 3 of Le Dizès (2000).

Once the code has $C\left(p_{j}\right)$ for each radius $r_{j}$, it may evaluate $\kappa\left(r_{j}\right)$, smooth it and step the mean profile $\bar{\omega}$ by applying the modified Crank-Nicolson scheme used for the model of Balmforth et al. (2001), with a no-flux condition for the vorticity at the origin and a zero vorticity boundary condition at $r=R$. For the simulations shown in figures 2 and 3, parameter values used are $(N, M, R, h)=(4000,24 N, 10, R / M)$ and figures $2(a)$ and $3(a) \Delta t=10^{-4}$ and figures $2(b, c)$ and $3(b, c) 10^{-5}$.

\section{Appendix C. Instability in the model of Balmforth et al. (2001)}

We initially ran the code for the model with no smoothing $\delta=0$ and found that the results depend strongly on numerical method, time-step and grid resolution. We 
were led to consider the evolution of weak, small scale perturbations on a profile and to what extent these may be amplified or suppressed by their effect on $\kappa$. Consider a smooth mean profile $\bar{\zeta}(y)$ which is perturbed to

$$
\bar{\zeta}(y)+\Delta \bar{\zeta}(y) \equiv \bar{\zeta}(y)+\mu \sin k y,
$$

with $\mu \ll 1$ and $k \gg 1$. We linearize, retaining only terms of order $\mu$, and it is convenient to use $\Delta$ to denote the change in a quantity to this order. The perturbation above will satisfy

$$
\partial_{\tau} \Delta \bar{\zeta}=\Delta \kappa \partial_{y}^{2} \bar{\zeta}+\kappa \partial_{y}^{2} \Delta \bar{\zeta}+\left(\partial_{y} \Delta \kappa\right)\left(1+\partial_{y} \bar{\zeta}\right)+\left(\partial_{y} \kappa\right)\left(\partial_{y} \Delta \bar{\zeta}\right)
$$

Now we have from (3.12),

$$
\Delta L_{\mathrm{r}}(p)=\pi \mu k \sin k p / m, \quad \Delta L_{\mathrm{i}}(p)=-\pi \mu k \cos k p / m,
$$

and also with $p \equiv m y$ from (3.15)

$$
\Delta \kappa(y)=-m^{2}|M(p)|^{4} w(p)\left[-2\left(p-L_{\mathrm{r}}(p)\right) \Delta L_{\mathrm{r}}(p)+2 L_{\mathrm{i}}(p) \Delta L_{\mathrm{i}}(p)\right],
$$

which amounts to

$$
\Delta \kappa(y)=2 \pi \mu k m^{2}|M(m y)|^{4} w(m y)\left[\left(m y-L_{\mathrm{r}}(m y)\right) \sin k y+L_{\mathrm{i}}(m y) \cos k y\right] .
$$

Now consider the four terms on the right-hand side of (C2). The first term, say (C2-i), and the fourth term (C2-iv) are of order $k$ only, and can be neglected in comparison with terms of order $k^{2}$. The second term (C2-ii) is

$$
\kappa \partial_{y}^{2} \Delta \bar{\zeta}=-\mu k^{2} \kappa \sin k y,
$$

which is the usual diffusion of the perturbation, while the third term (C 2-iii) involves the leading, order $k^{2}$ quantity

$$
\partial_{y} \Delta \kappa \simeq 2 \pi \mu k^{2} m^{2}|M(m y)|^{4} w(m y)\left[\left(m y-L_{\mathrm{r}}(m y)\right) \cos k y-L_{\mathrm{i}}(m y) \sin k y\right] .
$$

Now plainly the $\cos k y$ term here gives wave motion, but the sinky term can give growth or decay. Combining terms from (C2-ii) and (C2-iii) gives the (real) growth rate of the mode as

$$
\gamma=-k^{2} \kappa-2 \pi k^{2} m^{2}|M(m y)|^{4} w(m y) L_{\mathrm{i}}(m y)\left(1+\partial_{y} \bar{\zeta}\right) .
$$

Using the definition of $\kappa$ from (3.15) and $L_{\mathrm{i}}$ from (3.12), this can finally be written in the form

$$
\gamma(y)=\frac{\kappa(y)^{2} k^{2}}{m^{2} w(m y)}\left[L_{\mathrm{i}}(m y)^{2}-\left(m y-L_{\mathrm{r}}(m y)\right)^{2}\right] .
$$

Thus we have a highly unstable behaviour, with wavenumber $k$ possessing an order $k^{2}$ growth rate if this quantity is positive, i.e. if $L_{\mathrm{i}}$ is sufficiently large. For the initial condition of a constant gradient $\bar{\zeta} \equiv 0$ we have $L_{\mathrm{r}}=0, L_{\mathrm{i}}=-\pi$, giving formula (3.18) with amplification of modes in the range $|y|<\pi / m$. Note that while the limitations of such a local theory of instability are well known, the calculation is instructive as a simple means to understand the behaviour seen numerically.

\section{REFERENCES}

Balmforth, N. J., Llewellyn Smith, S. G. \& Young, W. R. 2001 Disturbing vortices. J. Fluid Mech. 426, 95-133.

Bassom, A. P. \& Gilbert, A. D. 1998 The spiral wind-up of vorticity in an inviscid planar vortex. J. Fluid Mech. 371, 109-140. 
Bassom, A. P. \& Gilbert, A. D. 1999 The spiral wind-up and dissipation of vorticity and a passive scalar in a strained planar vortex. J. Fluid Mech. 398, 245-270.

Boffetta, G., del Castillo Negrete, D., López, C., Pucacco, G. \& Vulpiani, A. 2003 Diffusive transport and self-consistent dynamics in coupled maps. Phys. Rev. E 67 (026224), 1-11.

Briggs, R. J., Daugherty, J. D. \& Levy, R. H. 1970 Role of Landau damping in crossed-field electron beams and inviscid shear flow. Phys. Fluids 13, 421-432.

del Castillo Negrete, D. $2000 a$ Self-consistent dynamics in the single wave model. Physica A 280, 10-21.

del Castillo Negrete, D. $2000 \mathrm{~b}$ Self-consistent chaotic transport in fluids and plasmas. Chaos 10, $75-88$.

Dritschel, D. G. \& McIntyre, M. E. 2008 Multiple jets as PV staircases: the Phillips effect and the resilience of eddy-transport barriers. J. Atmos. Sci. 65, 855-874.

Garaud, P. 2001 Latitudinal shear instability in the solar tachocline. Mon. Not. R. Astron. Soc. 324, 68-76.

Hall, I. M., Bassom, A. P. \& Gilbert, A. D. 2003 The effect of fine structure on the stability of planar vortices. Eur. J. Mech. B/Fluids 22, 179-198.

Haynes, P. H., Poet, D. A. \& Shuckburgh, E. F. 2007 Transport and mixing in kinematically and dynamically consistent flows. J. Atmos. Phys. 64, 3640-3651.

Heimpel, M. \& Aurnou, J. 2007 Turbulent convection in rapidly rotating spherical shells: a model for equatorial and high latitude jets on Jupiter and Saturn. Icarus 187, 540-557.

Le Dizès, S. 2000 Non-axisymmetric vortices in two-dimensional flows. J. Fluid Mech. 406, 175-198.

Mariotti, A., Legras, B. \& Dritschel, D. G. 1994 Vortex stripping and the erosion of coherent structures in two-dimensional flows. Phys. Fluids 6, 3954-3962.

McIntyre, M. E. \& Palmer, T. N. 1984 The 'surf zone' in the stratosphere. J. Atmos. Terr. Phys. 46, 825-849.

Norton, W. A. 1994 Breaking Rossby waves in a model stratosphere diagnosed by a vortexfollowing coordinate system and a technique for advecting material contours. J. Atmos. Sci. 51, 654-673.

Rotvig, J. \& Jones, C. A. 2006 Multiple jets and bursting in the rapidly rotating convecting two-dimensional annulus model with nearly plane-parallel boundaries. J. Fluid Mech. 567, $117-140$.

Schecter, D. A., Dubin, D. H. E., Cass, A. C., Driscoll, C. F., Lansky, I. M. \& O’Neil, T. M. 2000 Inviscid damping of asymmetries on a 2-d vortex. Phys. Fluids 12, 2397-2412.

Scott, R. K. \& Polvani, L. M. 2007 Forced-dissipative shallow-water turbulence on the sphere and the atmospheric circulation of the giant planets. J. Atmos. Sci. 64, 3158-3176.

Shrira, V. I. \& SAzonov, I. A. 2001 Quasi-modes in boundary-layer-type flows. Part 1. Inviscid two-dimensional spatially harmonic perturbations. J. Fluid Mech. 446, 133-171.

Turner, M. R. \& Gilbert, A. D. 2007 Linear and nonlinear decay of cat's eyes in two-dimensional vortices, and the link to Landau poles. J. Fluid Mech. 593, 255-279.

Turner, M. R. \& Gilbert, A. D. 2009 Spreading of two-dimensional axisymmetric vortices exposed to a rotating strain field. J. Fluid Mech. 630, 155-177.

Waugh, D. W. \& Plumb, R. A. 1994 Contour advection with surgery: a technique for investigating fine scale structure in tracer transport. J. Atmos. Sci. 51, 530-540. 